\title{
Association of serum lipid parameters with the severity and onset of atopic dermatitis in children
}

Ju Hee Kim ${ }^{1}$, Seung Won Lee ${ }^{2}$, Dong Keon Yon ${ }^{1}$, Eun Kyo Ha ${ }^{3}$, Hye Mi Jee ${ }^{1}$, Myongsoon Sung ${ }^{4}$, Hyeon Jeong Sim ${ }^{5}$, Jung Won Yoon ${ }^{6}$, Sun Hee Choi ${ }^{7}$, Youn Ho Shin ${ }^{8}$, Seung In $\mathrm{Seo}^{9}$, Heysung Baek ${ }^{9}$, and Man Yong $\operatorname{Han}^{1}$

${ }^{1}$ CHA Bundang Medical Center

${ }^{2}$ Sejong University

${ }^{3}$ Kangnam Sacred Heart Hospital

${ }^{4}$ Soon Chun Hyang University Gumi Hospital

${ }^{5} \mathrm{CHA}$ Medical University

${ }^{6}$ Myongji Hospital

${ }^{7}$ Kyung Hee University School of Medicine

${ }^{8} \mathrm{CHA}$ Gangnam Medical Center

${ }^{9}$ Kangdong Sacred Heart Hospital

June 22, 2020

\begin{abstract}
Background The association between dyslipidemia and atopic dermatitis in children is unclear. This study investigated the association between dyslipidemia and atopic dermatitis in children by analysis of disease onset, risk factors, and disease severity. Methods Subset I examined 7 year-old children in elementary school $(\mathrm{n}=248)$ and Subset II was a retrospective long-term follow-up hospital based-study ( $\mathrm{n}=52,725)$ conducted from 1986 to 2016 that used propensity score matching. In the Subset I Study, total cholesterol (TC), high-density lipoprotein cholesterol (HDL-C), low-density lipoprotein cholesterol (LDL-C), triglycerides (TG) were determined, and the severity of atopic dermatitis was determined using SCORing Atopic Dermatitis (SCORAD). In the Subset II Study, the time of atopic dermatitis onset was determined for asymptomatic subjects whose TC levels were below or above $170 \mathrm{mg} / \mathrm{dL}$. Results Our Subset I Study indicated that children with atopic dermatitis $(\mathrm{n}=69$, $27.8 \%$ ) had significantly higher levels of TC and TG, and that disease severity had significant associations with high levels of TC and TG, and a low level of HDL-C. Our Subset II Study (1,722 with high TC and 6,735 with normal TC after propensity score matching) indicated the high TC group had a greater hazard ratio (HR) for the onset of atopic dermatitis (consensus-based HR: $2.47 ; 95 \%$ CI: $1.23,5.06, \mathrm{P}=0.012$ ) during 5 years. Conclusion An abnormal blood lipid profile in children is associated with the presence and severity of atopic dermatitis. The risk of atopic dermatitis onset was significantly greater with high levels of TC.
\end{abstract}

Association of serum lipid parameters with the severity and onset of atopic dermatitis in children

\section{Conflict of interest statement}

There are no financial or other issues that might lead to conflict of interest.

\section{Financial support}

This study was supported by a grant from the Seongnam Atopy Project by the Seongnam City Government, Republic of Korea. The funders had no role in the data analysis or in the decision to publish. The funding 
agencies had no role in the design or conduct of the study; collection, management, analysis, or interpretation of the data; preparation, review, or approval of the manuscript; or the decision to submit the manuscript for publication.

\section{ABSTRACT}

\section{Background}

The association between dyslipidemia and atopic dermatitis in children is unclear. This study investigated the association between dyslipidemia and atopic dermatitis in children by analysis of disease onset, risk factors, and disease severity.

\section{Methods}

Subset I examined 7 year-old children in elementary school $(\mathrm{n}=248)$ and Subset II was a retrospective long-term follow-up hospital based-study $(\mathrm{n}=52,725)$ conducted from 1986 to 2016 that used propensity score matching. In the Subset I Study, total cholesterol (TC), high-density lipoprotein cholesterol (HDL-C), low-density lipoprotein cholesterol (LDL-C), triglycerides (TG) were determined, and the severity of atopic dermatitis was determined using SCORing Atopic Dermatitis (SCORAD). In the Subset II Study, the time of atopic dermatitis onset was determined for asymptomatic subjects whose TC levels were below or above $170 \mathrm{mg} / \mathrm{dL}$.

\section{Results}

Our Subset I Study indicated that children with atopic dermatitis $(\mathrm{n}=69,27.8 \%)$ had significantly higher levels of TC and TG, and that disease severity had significant associations with high levels of TC and TG, and a low level of HDL-C. Our Subset II Study (1,722 with high TC and 6,735 with normal TC after propensity score matching) indicated the high TC group had a greater hazard ratio (HR) for the onset of atopic dermatitis (consensus-based HR: 2.47; 95\% CI: 1.23, 5.06, $P=0.012$ ) during 5 years.

\section{Conclusion}

An abnormal blood lipid profile in children is associated with the presence and severity of atopic dermatitis. The risk of atopic dermatitis onset was significantly greater with high levels of TC.

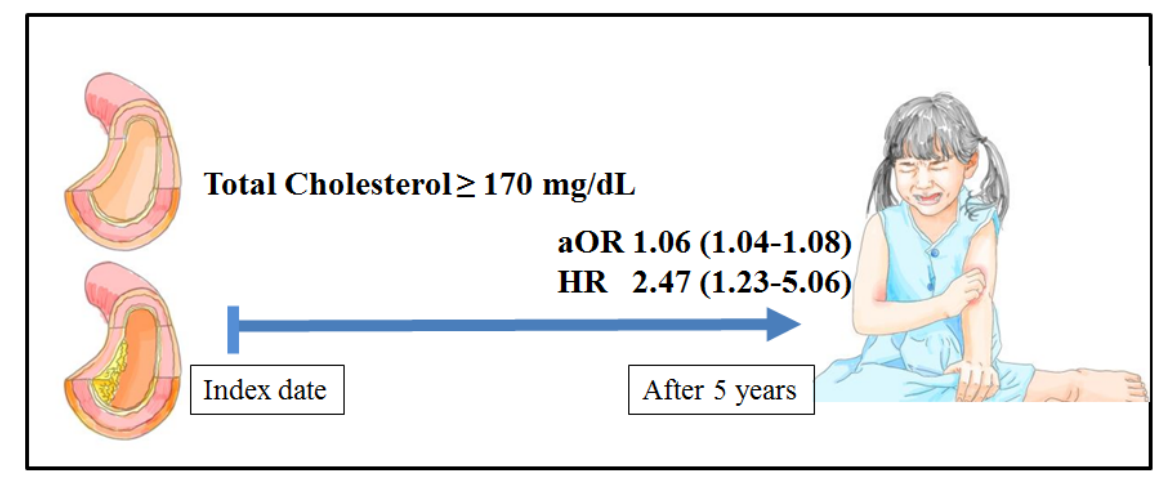

\section{Graphical Abstract}

KEYWORDS: dermatitis, atopic; cholesterol; dyslipidemias

\section{Introduction}

Atopic dermatitis is a complex disorder that is affected by genetic and environmental factors in which a defective skin barrier, reduced skin innate immune responses, and polarized adaptive immune responses to environmental allergens and microbes lead to chronic skin inflammation. ${ }^{1-3}$ Recent studies indicated that 
dyslipidemia is associated with an altered adaptive immune response of Th2 cells ${ }^{4}$ and the presence of allergic diseases, particularly allergen sensitization, ${ }^{5-7}$ asthma,,${ }^{6,8}$ and allergic rhinitis. ${ }^{9}$

However, only a limited number of studies have examined the lipid profiles of adults with atopic dermatitis. ${ }^{7,10-13}$ Some of these studies concluded that atopic dermatitis is associated with hyperlipidemia, ${ }^{7,10}$ but others reported no such association. ${ }^{11-13}$ In addition, few studies have examined the association of hyperlipidemia with atopic dermatitis in children, ${ }^{14}$ and no long-term follow up studies have directly assessed the severity of atopic dermatitis in children.

We hypothesized that children with atopic dermatitis have dyslipidemia. Thus, we examined one group of children and assessed severity of atopic dermatitis using SCORing Atopic Dermatitis (SCORAD) and measured the levels of total cholesterol (TC), high-density lipoprotein cholesterol (HDL-C), low-density lipoprotein cholesterol (LDL-C), and triglycerides (TG). We also hypothesized that dyslipidemia was a possible cause of atopic dermatitis, and performed a follow-up study of another group of asymptomatic children to record the onset of atopic dermatitis in those with different lipid profiles.

\section{Materials and Methods}

\section{Subset I study}

\section{Subjects}

A total of 618 children (7 years-old) from 11 randomly selected elementary schools in Seongnam City (Korea) were enrolled between June and July 2016. Each participant's parents were asked to respond to the questionnaires prior to a physical examination and blood sampling. 408 children (66.0\%) responded to the questionnaire and $305(74.8 \%)$ agreed to the blood test. Thus, 248 children who provided questionnaire data, skin prick test (SPT) results, and blood samples participated in the study.

\section{Study protocol}

Pediatric allergy specialists and trained field technicians performed the physical examinations, SCORAD score assessments, ${ }^{15} \mathrm{SPTs}$, and collected blood samples. The presence of current atopic dermatitis was diagnosed using the International Study of Asthma and Allergies in Childhood (ISAAC) questionnaire, ${ }^{16}$ based on symptoms during the previous 12 months. The study protocol was approved by the Institutional Review Board of CHA University (2016-04-031) and written informed consent documents were obtained from all parents or guardians of children who participated.

\section{SCORAD score}

Assessments of SCORAD scores (range: 0 to 83) were performed by 2 pediatricians who specialized in allergies and visited each school to perform physical examinations. The pediatricians were blinded to the questionnaire responses. Children were divided into three groups based on SCORAD score: mild $(<25)$, moderate (25 to 50$)$, or severe $(>50){ }^{15}$

\section{Measurement of blood lipid profile}

Venous non-fasting serum samples were obtained at scheduled visits in the clinic and TC, LDL-C, HDL-C, and TG were determined. ${ }^{17}$ Any child with a serum TC level over $170 \mathrm{mg} / \mathrm{dL}$, TG level over $75 \mathrm{mg} / \mathrm{dL}$, LDL-C level over $110 \mathrm{mg} / \mathrm{dL}$, or HDL-C level under $45 \mathrm{mg} / \mathrm{dL}$ was classified as having dyslipidemia. ${ }^{18}$

\section{Subset II study}

Study population and data collection using the Observational Medical Outcomes Partnership Common Data Model (OMOP-CDM)

A single-center long-term cohort study using the OMOP-CDM was used for a retrospective analysis of clinical data from Hallym University, Kangdong Sacred Heart Hospital, Seoul, South Korea. Patient-level data were transformed into a common data format that enabled identical study execution. De-identified data were from 
electronic medical records and were transformed into the OMOP-CDM format. ${ }^{19}$ The Institutional Review Board of Hallym University provided approval for the analysis (IRB 2019-09-005).

\section{Conversion of Data to the OMOP CDM}

The OMOP-CDM structures and harmonizes patient-level data, including details of visits with health care services, diagnoses, medical procedures, drugs, and laboratory test results, and de-identifies clinical content. A diagnosis record consists of a patient identifier, the date of diagnosis, a code for the diagnosis, and the mapping and coding system (International Classification of Diseases, Tenth Revision [ICD-10]) to the OMOPCDM Standardized Vocabularies. ${ }^{20}$ In this mapping process, the Systematic Nomenclature of Medicine (SNOMED) is used as the target vocabulary for diagnostic codes, RxNorm for drugs, and Logical Observation Identifiers Names and Codes for other observations, such as laboratory results and measurements of vital signs. ${ }^{19}$

\section{Cohort construction}

Children less than 18 years-old were enrolled, and the day of the blood draw for cholesterol measurements was defined as the index date. Subjects with atopic dermatitis diagnosis codes or prescriptions for treatment of atopic dermatitis before the index date were excluded. Children were also excluded if they had previous recorded diagnoses of contact dermatitis, psoriasis, scabies, ichthyosis, erythroderma, photodermatitis, or seborrheic dermatitis. Subjects were separated into a high TC group and a low TC group according to a blood TC level of $170 \mathrm{mg} / \mathrm{dL} .{ }^{21}$ The propensity model and outcome definitions all operate on data that are converted into the CDM. ${ }^{19}$ Thus, there were matching for age, sex, medications, procedures, and measurements based on the medical records of patients in the high and normal TC groups. The association of TC with the onset of atopic dermatitis was assessed using medical data from Kandong Sacred Heart Hospital. The unmatched cohort had 3,913 children with TC levels of $170 \mathrm{mg} / \mathrm{dL}$ or more (high TC group) and 48,812 children with TC levels below $170 \mathrm{mg} / \mathrm{dL}$ (normal TC group). After 1:4 propensity score matching, the matched cohort had 1,722 subjects in the high TC group and 6,735 subjects in the normal TC group.

\section{Outcomes}

The primary outcome was the onset of atopic dermatitis based on Logical Observation Identifiers Names and Codes, in which codes were mapped to their corresponding SNOMED codes. ${ }^{19}$ To accurately identify patients with atopic dermatitis in the electronic health records, atopic dermatitis was defined as 5 diagnoses based on the ICD-10 codes for atopic dermatitis (L20.*) and 2 treatment codes for atopic dermatitis drug therapy. ${ }^{22}$ Atopic dermatitis drug therapy was defined as topical therapy, such as corticosteroids, calcineurin inhibitors, and a phosphodiesterase inhibitor. ${ }^{1,22}$

\section{Statistical analysis}

All statistical analyses were performed using IBM SPSS Statistics 25.0 (IBM Corp., NY, USA). Continuous data were expressed as means with standard deviations or medians with interquartile ranges (depending on data distribution). The Mann-Whitney $U$ test was used to compare continuous variables and a posthoc analysis with the Dunn-test and the chi-square test were used to compare categorical variables. After adjustment for gender and body mass index (BMI), correlations between lipid level and the SCORAD score were evaluated by partial correlation analysis. Generalized linear models with the logit function or gamma function (depending on data distribution) were used to determine the odds ratios or beta values for the relationship between lipid parameters and atopic dermatitis or SCORAD score.

Propensity scores were used to mitigate biases arising from nonrandom treatment assignment. Thus, for each pairwise comparison, matched cohorts were constructed using 1:4 propensity score matching with a caliper of 0.25 on the logit scale. ${ }^{23,24}$ A Cox proportional hazard model was then fit to the matched cohorts using the Cohort Method R package (https://github.com/OHDSI/CohortMethod), and the hazard ratio (HR) and $95 \%$ confidence interval (CI) was calculated for each outcome of interest, a procedure that reduces residual bias and variance. ${ }^{25}$ 


\section{Sensitivity Analyses}

To assess the robustness of the results, several Cox proportional hazard analyses were also performed. First, a less stringent definition of atopic dermatitis was used, in which atopic dermatitis was defined as more than 1 ICD-10 diagnostic code and at least 2 atopic dermatitis-related treatments. A previous study reported that the presence of at least 1 of 5 diagnostic codes plus 2 treatment codes for any skin-directed therapy had a positive predictive value (PPV) of $86 \%$ (95\% CI $=80$ to 91) for physician-confirmed diagnosis of atopic dermatitis. ${ }^{22}$ Second, a more stringent definition was used, in which hyperlipidemia was defined as a TC level above $200 \mathrm{mg} / \mathrm{dL}$. This definition excludes those with borderline-high TC levels (170 to $199 \mathrm{mg} / \mathrm{dL}$ ).

\section{Results}

\section{Characteristics of study subjects: Subset I Study}

Our Subset I Study examined 248 children, 179 in a control group and 69 in an atopic dermatitis group (Table 1). These two groups had similar age, height, BMI, and gender distribution, and no significant differences in the rates of asthma, allergic rhinitis, passive smoking exposure, prematurity, low birthweight, or allergic sensitization.

Analysis of blood lipid levels (Figure 1, Table 1) indicated that children with atopic dermatitis had significantly higher mean levels of TC (173.0 [95\% CI: 158.0, 191.0] vs. 194.0 [95\% CI: $188.0,205.5] \mathrm{mg} / \mathrm{dL}, P$ $<0.001)$ and TG $(76.0$ [95\% CI: 55.0, 107.0] vs. 97.0 [95\% CI: $65.0,129.5] \mathrm{mg} / \mathrm{dL}, P=0.006)$, but the two groups had similar levels of LDL-C and HDL-C.

\section{Lipid parameters and SCORAD score: Subset I Study}

The SCORAD score was significantly higher in the atopic dermatitis group than in the control group (2.49 [95\% CI: 0.00, 0.00] vs.17.0 [95\% CI: 0.00, 27.30], $P=0.001$ ). Among children with atopic dermatitis, the SCORAD score indicated mild disease (SCORAD $<25)$ in 20 children $(29.0 \%)$ and moderate-severe disease (SCORAD [?] 25) in 22 children (31.96\%). Analysis of SCORAD score and blood lipid profiles in all 248 subjects (Table 2) indicated that children with moderate-severe disease had significantly higher levels of TC and TG and lower levels of HDL-C than control children and children with mild disease.

\section{Lipid parameters, atopic dermatitis, and SCORAD score: Subset I Study}

Adjustment for confounding by gender and BMI z score (Table 3) indicated the aOR for atopic dermatitis was greater in children with higher levels of TC (aOR: 1.057, 95\% CI: 1.037, 1.076, $P<0.001$ ) and TG (aOR: $1.009,95 \%$ CI: $1.003,1.014, P=0.004$ ). The SCORAD score also had a positive association with the levels of TC ( $\beta$ coefficient: $0.085 ; 95 \%$ CI: $0.021,1.150 ; P=0.010)$ and TG ( $\beta$ coefficient: $0.085 ; 95 \%$ CI: $0.021,1.150 ; P=0.006)$, but a negative association with HDL-C level ( $\beta$ coefficient: $-0.151 ; 95 \%$ CI: -0.260 to $-0.043 ; P=0.006)$.

\section{TC and onset of atopic dermatitis: Subset II Study}

Our Subset II Study examined the onset of atopic dermatitis in children during a median follow-up time of 1,750 days (Figure 2). Among 155 subjects with high TC, 21 (13.5\%) had new onset atopic dermatitis; and among 863 subjects with normal TC, $48(5.6 \%)$ had new onset atopic dermatitis. The results indicated a significantly increased probability of atopic dermatitis in the high TC group (Hazard Ratio [HR]: 2.47; 95\% CI: $1.23,5.06 ; P=0.012)$. The results of sensitivity analysis, in which we used more stringent criteria to define high TC (Supplementary Figure E1) and less stringent criteria to define atopic dermatitis (Supplementary Figure E2) were consistent.

\section{Discussion}

Our Subset I Study indicated that a high TC level correlated with the severity of atopic dermatitis in schoolage children, and our Subset II Study (a single center long-term study using OMOP-CDM) indicated that 
the high TC group had a slightly greater HR for the onset of atopic dermatitis than the normal TC group. Several previous studies examined the relationship of hyperlipidemia with atopic dermatitis in adults.7,10-14 The results of the present study of children are consistent that reported a positive correlation of hyperlipidemia with atopic dermatitis in adults. ${ }^{7,10}$ However, very few studies have examined the association of atopic dermatitis with lipid parameters in children. A database study by Augustin et al. ${ }^{14}$ showed a significantly increased prevalence rate of hyperlipidemia in children with atopic dermatitis compared to those without atopic dermatitis (prevalence ratio: 1.29; $95 \%$ CI: 1.12, 1.49; $P<0.05$ ). However, their study defined atopic dermatitis based only on ICD code, did not directly measure TC, LDL-C, HDL-C, or TG, did not report the severity of atopic dermatitis, and was a cross-sectional study.

Previous research indicated that dyslipidemia is a type of chronic inflammatory response. ${ }^{26}$ In particular, elevated TG and LDL-C lead to increased pro-inflammatory signaling and increased expression of TNF- $\alpha$ and interleukin (IL)-6. ${ }^{26,27}$ In contrast, HDL-C has an anti-inflammatory effect, in that it modulates Tcell activation. ${ }^{26,27}$ This supports the interpretation that the presence of a chronic inflammatory state (as indicated by dyslipidemia) is responsible for chronic skin inflammation, and provides a possible mechanism for the relationship between atopic dermatitis and hyperlipidemia.

We used the SCORAD score to grade the severity of atopic dermatitis, and found that this score had positive associations with TC and TG and a negative association with HDL-C. Thus, our results indicate an association of hyperlipidemia with the severity of atopic dermatitis. However, another previous case-control study of adults in Germany reported conflicting results in the relationship of dermatitis with HDL-C. ${ }^{7}$ This study found that the HDL-C level was significantly higher in subjects with atopic dermatitis $(61.0$ vs.54.9 $\mathrm{mg} / \mathrm{dL} ; P<0.01)$ and that after stratification by gender, there was a significant positive association of HDL level and atopic dermatitis in men. ${ }^{7}$ Our results indicated that disease severity (SCORAD score), erythema, excoriation, lichenification, and pruritus scores in children had positive correlations with the levels of TC and TG, and negative correlations with HDL-C level. Thus, cholesterol might be related to the chronicity of atopic dermatitis in children.

The results of our Subset II Study indicated that the high TC group had a greater HR for the onset of atopic dermatitis during a median follow-up time of 1,750 days. The Observational Health Data Sciences and Informatics (OHDSI) initiative is a large-scale international collaborative that is investigating the value of analyzing health data. ${ }^{20}$

We used a retrospective long-term follow-up study (Subset II) to complement our cross-sectional study (Subset I) and examine the casual relationship of hyperlipidemia and atopic dermatitis. Nonetheless, there were several limitations to this study. We did not perform repeated objective clinical assessments and there are data quality issues inherent to working with health care databases, in that covariates, exposures, and outcomes may be inadequately or incorrectly measured. However, our use of data standardization into a common data model, propensity-score matching, and calibration via negative controls helped to prevent erroneous conclusions. Also, the case definition of atopic dermatitis was based on ICD-10 diagnostic codes, so there may be questions about the validity of our definition. To address this issue, we defined atopic dermatitis as at least 5 diagnoses of atopic dermatitis from ICD codes plus 2 treatment codes for atopic dermatitis drug therapy. ${ }^{22}$

Our study has many merits. To the best of our knowledge, this is the first large-sample study of urban children to comprehensively investigate the association between blood lipid level and the severity of atopic dermatitis. Moreover, trained physicians directly assessed the severity of atopic dermatitis. Most previous studies of the association between atopic dermatitis and hyperlipidemia were surveys, and none of these previous studies examined the disease severity. In our study, pediatricians specializing in allergies determined all SCORAD scores. Another strength is a longitudinal cohort analysis to supplement and valify the cross-sectional analysis.

In conclusion, our results indicated that hyperlipidemia in children has a positive association with the presence and severity of atopic dermatitis. These findings suggest that atopic dermatitis should be considered 
a systemic disorder that shares certain characteristics with other chronic inflammatory disorders.

\section{Author contributions}

Ju Hee Kim, Seung Won Lee, Hey Sung Baek and Man Young Han contributed to conception and design; Seung Won Lee, Dong Gun Yeon, Hye Mi Jee and Man Young Han acquired the data; Man Young Han, Ju Hee Kim, Seung Won Lee, Eun Kyo Ha, Youn Ho Shin, Seoung In Seo, Sun Hee Choi, Myungsoon Seong analyzed and interpreted the data; Ju Hee Kim and Seung Won Lee drafted the article; Dong Keon Yon, Seung Won Lee, Jung Won Yoon, Hyun Jung Shim and Youn Ho Shin critically revised the article and provided important intellectual content; and all authors approved the final version to be published.

\section{Acknowledgments}

We are grateful to the children and families who participated in the study for their support and dedication. We thank the Department of Environment Policy at Seongnam City Government for its assistance and cooperation.

\section{Impact Statement}

Association between atopic dermatitis and dyslipidemia in children is a matter of controversy. In addition, there is a paucity of studies with longitudinal follow up regarding the onset of atopic dermatitis in children with dyslipidemia. The presence and severity of atopic dermatitis in children is associated with abnormal blood lipid profiles. Also, subjects with high TC levels are in higher risks of developing atopic dermatitis. Our study suggests that children with dyslipidemia may be need close monitoring for the development of atopic dermatitis. Furthermore, our results imply that cholesterol management might be able to lower the risk of atopic dermatitis.

\section{References}

1. Jo SY, Lee C-H, Jung W-J, Kim S-W, Hwang Y-H. Common features of atopic dermatitis with hypoproteinemia. Clin Exp Pediatr.2018;61(11):348-354.

2. Kennedy K, Heimall J, Spergel JM. Advances in atopic dermatitis in 2017. Journal of Allergy and Clinical Immunology.2018;142(6):1740-1747.

3. Ha J, Lee SW, Yon DK. 10-year trends and prevalence of asthma, allergic rhinitis, and atopic dermatitis among the Korean population, 2008-2017. J Korean Pediatr Soc. 2020;0(0):0-0.

4. Robertson AK, Zhou X, Strandvik B, Hansson GK. Severe hypercholesterolaemia leads to strong Th2 responses to an exogenous antigen. Scand J Immunol. 2004;59(3):285-293.

5. Ouyang F, Kumar R, Pongracic J, et al. Adiposity, serum lipid levels, and allergic sensitization in Chinese men and women. J Allergy Clin Immunol. 2009;123(4):940-948 e910.

6. Vinding RK, Stokholm J, Chawes BLK, Bisgaard H. Blood lipid levels associate with childhood asthma, airway obstruction, bronchial hyperresponsiveness, and aeroallergen sensitization. J Allergy Clin Immunol. 2016;137(1):68-74 e64.

7. Schafer T, Ruhdorfer S, Weigl L, et al. Intake of unsaturated fatty acids and HDL cholesterol levels are associated with manifestations of atopy in adults. Clin Exp Allergy. 2003;33(10):1360-1367.

8. Peng J, Huang Y. Meta-analysis of the association between asthma and serum levels of high-density lipoprotein cholesterol and low-density lipoprotein cholesterol. Ann Allergy Asthma Immunol.2017;118(1):6165.

9. Yon DK, Lee SW, Ha EK, et al. Serum lipid levels are associated with allergic rhinitis, nasal symptoms, peripheral olfactory function, and nasal airway patency in children. Allergy. 2018;73(9):1905-1908.

10. Silverberg JI, Greenland P. Eczema and cardiovascular risk factors in 2 US adult population studies. $J$ Allergy Clin Immunol.2015;135(3):721-728 e726. 
11. Megna M, Patruno C, Balato A, et al. An Italian multicentre study on adult atopic dermatitis: persistent versus adult-onset disease. Arch Dermatol Res. 2017;309(6):443-452.

12. Standl M, Tesch F, Baurecht H, et al. Association of Atopic Dermatitis with Cardiovascular Risk Factors and Diseases. J Invest Dermatol. 2017;137(5):1074-1081.

13. Lee JH, Jung HM, Han KD, et al. Association Between Metabolic Syndrome and Atopic Dermatitis in Korean Adults. Acta Derm Venereol. 2017;97(1):77-80.

14. Augustin M, Radtke MA, Glaeske G, et al. Epidemiology and Comorbidity in Children with Psoriasis and Atopic Eczema.Dermatology. 2015;231(1):35-40.

15. Oranje AP, Glazenburg EJ, Wolkerstorfer A, de Waard-van der Spek FB. Practical issues on interpretation of scoring atopic dermatitis: the SCORAD index, objective SCORAD and the three-item severity score.Br J Dermatol. 2007;157(4):645-648.

16. Lee SI, Shin MH, Lee HB, et al. Prevalences of symptoms of asthma and other allergic diseases in korean children: a nationwide questionnaire survey. J Korean Med Sci. 2001;16(2):155-164.

17. Kim KE, Baek KS, Han S, Kim JH, Shin YH. Serum alanine aminotransferase levels are closely associated with metabolic disturbances in apparently healthy young adolescents independent of obesity. Korean $J$ Pediatr. 2019;62(2):48-54.

18. Peterson AL, McBride PE. A review of guidelines for dyslipidemia in children and adolescents. WMJ. 2012;111(6):274-281; quiz 282.

19. FitzHenry F, Resnic F, Robbins S, et al. Creating a common data model for comparative effectiveness with the observational medical outcomes partnership. Applied clinical informatics.2015;6(03):536-547.

20. Hripcsak G, Duke JD, Shah NH, et al. Observational Health Data Sciences and Informatics (OHDSI): opportunities for observational researchers. Studies in health technology and informatics.2015;216:574.

21. FOR EPOIG, CHILDREN RRI. Expert panel on integrated guidelines for cardiovascular health and risk reduction in children and adolescents: summary report. Pediatrics. 2011;128(Suppl 5):S213.

22. Abuabara K, Magyari AM, Hoffstad O, et al. Development and validation of an algorithm to accurately identify atopic eczema patients in primary care electronic health records from the UK. Journal of Investigative Dermatology. 2017;137(8):1655-1662.

23. Stuart EA. Matching methods for causal inference: A review and a look forward. Stat Sci. 2010;25(1):121.

24. Austin PC. Optimal caliper widths for propensity-score matching when estimating differences in means and differences in proportions in observational studies. Pharm Stat. 2011;10(2):150-161.

25. Essert C, Haegelen C, Lalys F, Abadie A, Jannin P. Automatic computation of electrode trajectories for Deep Brain Stimulation: a hybrid symbolic and numerical approach. Int J Comput Assist Radiol Surg. $2012 ; 7(4): 517-532$.

26. Welty FK, Alfaddagh A, Elajami TK. Targeting inflammation in metabolic syndrome. Translational research. 2016;167(1):257-280.

27. Manti S, Leonardi S, Panasiti I, Arrigo T, Salpietro C, Cuppari C. Serum IL-10, IL-17 and IL-23 levels as "bioumoral bridges" between dyslipidemia and atopy. Cytokine. 2017;99:43-49.

Table 1. Demographic and clinical characteristics of children enrolled in the subset I study $(\mathrm{n}=248)$.

\begin{tabular}{lll}
\hline Control & Atopic dermatitis & $P$ value \\
\hline$(\mathrm{n}=179,72.2 \%)$ & $(\mathrm{n}=69,27.8 \%)$ &
\end{tabular}




\begin{tabular}{|c|c|c|c|}
\hline & Control & Atopic dermatitis & $P$ value \\
\hline \multicolumn{4}{|l|}{ Demographics } \\
\hline Age, years, mean (SD) & $7.10(0.30)$ & $7.07(0.29)$ & 0.595 \\
\hline Sex, male, n $(\%)$ & $100(55.9)$ & $38(55.1)$ & 0.910 \\
\hline \multicolumn{4}{|l|}{ Anthropometrics } \\
\hline BMI z score, n (SD) & $0.07(1.06)$ & $-0.10(1.16)$ & 0.274 \\
\hline Height, cm, n (SD) & $121.5(5.3)$ & $121.7(5.3)$ & 0.800 \\
\hline Obesity $^{*}, \mathrm{n}(\mathrm{SD})$ & $62(28.1)$ & $4(22.2)$ & 0.595 \\
\hline \multicolumn{4}{|c|}{ Lipid profile, median $[\mathrm{IQR}]$} \\
\hline $\mathrm{TC}, \mathrm{mg} / \mathrm{dL}$ & $137.0[158.0-191.0]$ & $194.0[188.0-205.5]$ & $<0.01$ \\
\hline $\mathrm{TG}, \mathrm{mg} / \mathrm{dL}$ & $76.0[55.0-107.0]$ & $97.0[65.0-129.5]$ & 0.006 \\
\hline LDL-C, mg/dL & $96.0[83.0-112.0]$ & $90.0[79.5-105.5]$ & 0.172 \\
\hline HDL-C, mg/dL & $62.0[54.0-75.0]$ & $61.0[52.0-69.5]$ & 0.112 \\
\hline \multicolumn{4}{|l|}{ Dyslipidemia } \\
\hline \multicolumn{4}{|l|}{$\mathrm{TC}, \mathrm{n}(\%)$} \\
\hline Normal & $84(33.9)$ & $0(0.0)$ & $<0.01$ \\
\hline Abnormal & $95(38.3)$ & $69(27.8)$ & \\
\hline \multicolumn{4}{|l|}{$\mathrm{TG}, \mathrm{n}(\%)$} \\
\hline Normal & $86(34.7)$ & $22(8.9)$ & 0.021 \\
\hline Abnormal & $93(37.5)$ & $47(19.0)$ & \\
\hline \multicolumn{4}{|l|}{ LDL-C, n (\%) } \\
\hline Normal & $130(52.4)$ & $54(21.8)$ & 0.363 \\
\hline Abnormal & $49(19.8)$ & $15(6.0)$ & \\
\hline \multicolumn{4}{|l|}{ HDL-C, n (\%) } \\
\hline Normal & $174(70.2)$ & $64(25.8)$ & 0.110 \\
\hline Abnormal & $5(2.0)$ & $5(2.0)$ & \\
\hline
\end{tabular}

Abbreviations: SD, standard deviation; BMI, body mass index; LDL-C, Low-density lipoprotein cholesterol; HDL-C, High-density lipoprotein cholesterol; TG, Triglyceride; TC, Total cholesterol;

*Obesity definition was BMI z score [?]1.96.

Missing data in BMI z score, $\mathrm{n}=8$; height, $\mathrm{n}=9$; and obesity, $\mathrm{n}=8$.

Total cholesterol levels $>170 \mathrm{mg} / \mathrm{dL}$, triglyceride levels $>75 \mathrm{mg} / \mathrm{dL}$, low-density lipoprotein cholesterol levels $>110 \mathrm{mg} / \mathrm{dL}$, or high-density lipoprotein cholesterol levels $<45 \mathrm{mg} / \mathrm{dL}$ were taken as abnormal.

Table 2. Relationship of SCORAD score with lipid parameters in the subset I study.

\begin{tabular}{lllll}
\hline & Severity of SCORAD & Severity of SCORAD & Severity of SCORAD & \\
\hline & Healthy control & Mild SCORAD & Mod-Severe SCORAD & $P$ value \\
Total cholesterol, median [IQR] & $180.5[158.0-195.0]$ & $184.0[168.0-197.0]$ & $\mathbf{1 9 0 . 5}[\mathbf{1 8 1 . 8}-\mathbf{1 9 8 . 8}]^{*}$ & $\mathbf{0 . 0 4 8}$ \\
Normal, n (\%) & $69(83.1)$ & $11(13.3)$ & $3(3.6)$ & $\mathbf{0 . 0 1 1}$ \\
Abnormal, n (\%) & $109(66.5)$ & $32(19.5)$ & $23(14.0)$ & \\
Triglyceride, median [IQR] & $75.5[56.8-104.0]$ & $86.0[60.0-126.0]$ & $\mathbf{1 0 7 . 0}[\mathbf{6 0 . 5}-\mathbf{1 4 5 . 5}]^{*}$ & $\mathbf{0 . 0 0 8}$ \\
Normal, n (\%) & $86(80.4)$ & $14(13.1)$ & $7(6.5)$ & $\mathbf{0 . 0 3 5}$ \\
Abnormal, n (\%) & $92(65.7)$ & $29(20.7)$ & $19(13.6)$ & 0.081 \\
LDL-C, median [IQR] & $96.0[83.0-112.0]$ & $96.0[81.0-106.0]$ & $87.0[74.8-95.3]$ & 0.35 \\
Normal, n (\%) & $128(69.9)$ & $33(18.0)$ & $22(12.0)$ & \\
Abnormal, n (\%) & $50(78.1)$ & $10(15.6)$ & $4(6.3)$ & $\mathbf{0 . 0 0 3}$ \\
HDL-C, median [IQR] & $65.0[54.8-76.0]$ & $58.0[53.0-70.0]$ & $\mathbf{5 6 . 5}[\mathbf{5 1 . 8}-\mathbf{6 2 . 3}]^{*}$ & $\mathbf{0 . 0 0 3}$
\end{tabular}




\begin{tabular}{lllll}
\hline & Severity of SCORAD & Severity of SCORAD & Severity of SCORAD & 0.117 \\
\hline Normal, n $(\%)$ & $172(72.6)$ & $42(17.7)$ & $23(9.7)$ & \\
Abnormal, n $(\%)$ & $6(60.0)$ & $1(10.0)$ & $3(30.0)$ & \\
\hline
\end{tabular}

Abbreviations: IQR, interquartile range; LDL-C, Low-density lipoprotein cholesterol; HDL-C, High-density lipoprotein cholesterol.

All continuous variables were analyzed using the nonparametric Mann-Whitney U test.

${ }^{*} P$ value $<0.05$ compared to healthy controls using post-hoc analysis with Dunn test.

Total cholesterol levels $>170 \mathrm{mg} / \mathrm{dL}$, triglyceride levels $>75 \mathrm{mg} / \mathrm{dL}$, low-density lipoprotein cholesterol levels $>110 \mathrm{mg} / \mathrm{dL}$, or high-density lipoprotein cholesterol levels $<45 \mathrm{mg} / \mathrm{dL}$ were taken as abnormal.

The SCORAD score: mild $(<25)$, moderate $(25-50)$, and severe $(>50)$.

Table 3. Relationship of atopic dermatitis and SCORAD score with lipid parameters in the subset I study.

\begin{tabular}{|c|c|c|c|c|}
\hline & Atopic dermatitis & Atopic dermatitis & Atopic dermatitis & Atopic dermatitis \\
\hline Lipid profile & OR $(95 \% \mathrm{CI})$ & $P$ value & $\mathrm{aOR}(95 \% \mathrm{CI})^{*}$ & $P$ value \\
\hline Total cholesterol & $1.053(1.035-1.071)$ & $<0.001$ & $1.057(1.037-1.076)$ & $<0.01$ \\
\hline Triglyceride & $1.008(1.002-1.013)$ & 0.007 & $1.009(1.003-1.014)$ & 0.004 \\
\hline LDL-C & $0.993(0.980-1.006)$ & 0.277 & $0.992(0.979-1.005)$ & 0.240 \\
\hline HDL-C & $0.985(0.965-1.005)$ & 0.984 & $0.985(0.964-1.005)$ & 0.143 \\
\hline
\end{tabular}

Abbreviations: CI, Confidence interval; OR, Odds ratio; LDL-C, Low-density lipoprotein cholesterol; HDLC, High-density lipoprotein cholesterol.

${ }^{*}$ Generalized linear models with the logit or gamma function, depending on the data distribution, were used to determine the odds ratio or beta value between lipid profiles and atopic dermatitis presence or SCORAD score adjusted for gender, and BMI z score.

\section{Figure legends}

Figure 1. Relative to controls, children with $\mathrm{AD}$ had significantly higher levels of total cholesterol (A; 173.0 $[158.0,191.0]$ vs. $194.0[188.0,205.5] \mathrm{mg} / \mathrm{dL}, \mathrm{P}<0.001)$ and total triglycerides $(\mathrm{B} ; 76.0[55.0,107.0)$ vs. 97.0 $[65.0,129.5] \mathrm{mg} / \mathrm{dL}, \mathrm{P}=0.006)$, but these two groups had no statistically significant differences in the levels of LDL-C (C; $96.0[83.0,112.0)$ vs. $90.0[79.5,105.5] \mathrm{mg} / \mathrm{dL}, \mathrm{P}=0.172)$ and HDL-C (D; 62.0 [54.0, 75.0] vs. $61.0[52.0,69.5] \mathrm{mg} / \mathrm{dL}, \mathrm{P}=0.172)$. Red dashed lines indicate borders between normal and abnormal values.

Figure 2. Kaplan-Meier curves for the probability of disease-free survival (no atopic dermatitis) in children with normal total cholesterol (blue line) and high total cholesterol (red line). Criteria used to define atopic dermatitis: at least 5 diagnostic codes based on ICD-10, and total cholesterol $>170 \mathrm{mg} / \mathrm{dL}$. Consensus hazard ratio: 2.47; 95\% CI: 1.23, 5.06, P = 0.012. Abbreviations: CI, Confidence interval; HR, hazard ratio; Ref, Reference. 

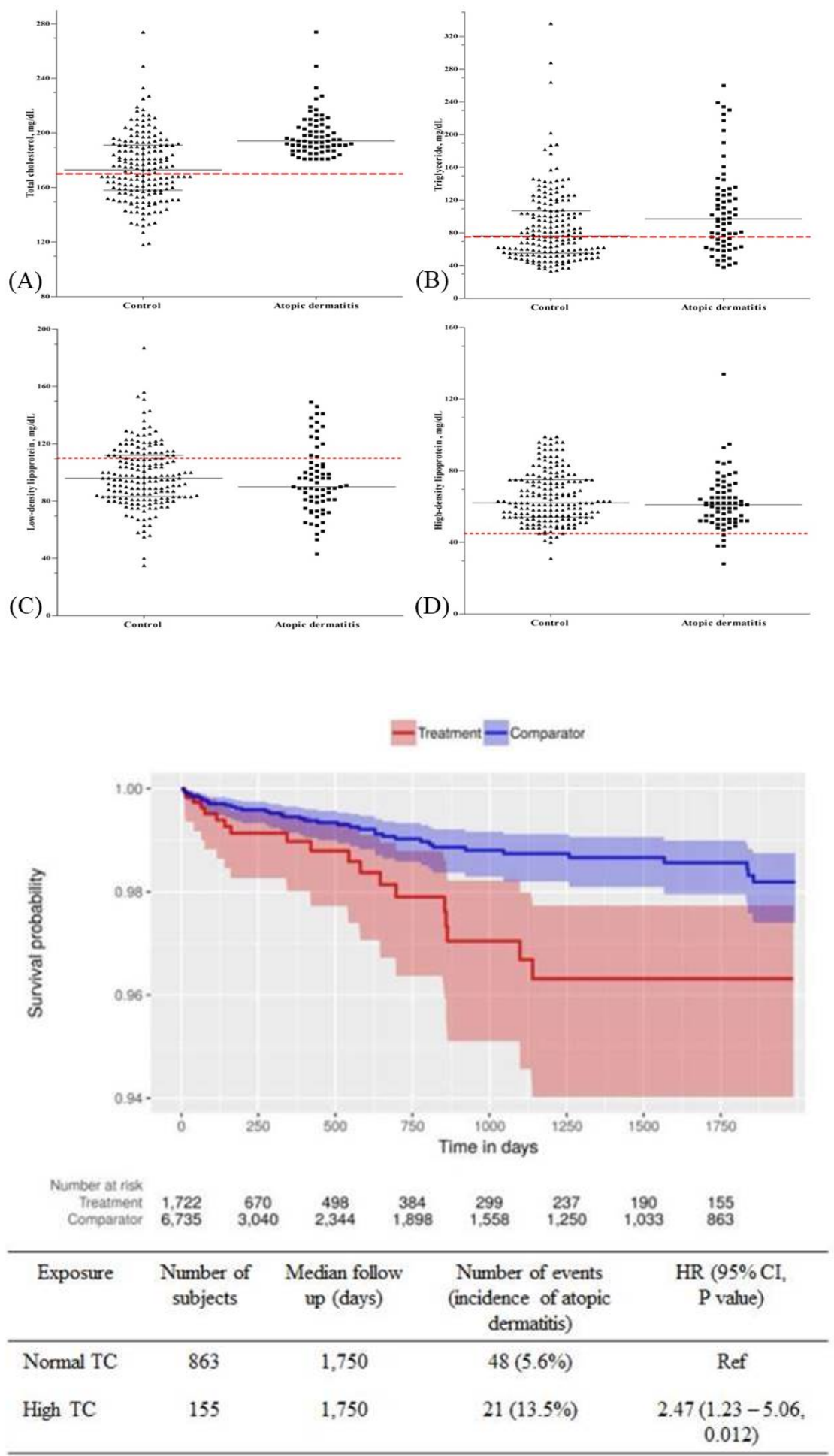